Disponível em:

http://editora.unoesc.edu.br/index.php/race

Race, Joaçaba, v. 15, n. 1, p. 251-274, jan./abr. 2016

\title{
CONEXÃO VISUAL E EMOCIONAL E A INTENÇÃO DE COMPRAR LINGERIE PARA MULHERES E A MOD̉ERAÇÃO DO SEU ESTADO CIVIL
}

\section{Visual and emotional connection and the intention of buying lingerie for women and marital status moderation}

Rafael Mendes Lübeck

E-mail: rafael.lubeck@gmail.com

Mestre em Administração pela Universidade Federal de Santa Maria; doutorando em Administração, linha de pesquisa Marketing na Pontíficia Universidade Católica do

Rio Grande do Sul.

\begin{abstract}
Aline Hopner
E-mail: alinehop@yahoo.com.br

Mestre em Administração pela Universidade Federal do Rio Grande do Sul; doutoranda em Administração pela Pontifícia Universidade Católica do Rio Grande do
\end{abstract}

Fernando Oliveira Santini

E-mail: santiniconsultores@terra.com.br

Doutor em Administração pela Pontifica Universidade Católica do Rio Grande do Sul; Mestre em Administração e Negócios pela Pontifícia Universidade Católica do

Rio Grande do Sul; Professor da Universidade do Vale do Rio dos Sinos e Escola Superior de Propaganda e Marketing . Endereço para correspondência: Avenida Unisinos, 950, Cristo Rei, 93022-000, São Leopoldo, Rio Grande do Sul, Brasil.

Joao Paulo Capelli Martins

E-mail: jpcapell@terra.com.br Mestre em Administração pela Universidade Federal do Rio Grande do Sul; doutorando em Administração pela Pontifícia Universidade Católica do Rio Grande do 
Artigo recebido em 07 de maio de 2015. Aceito em 30 de novembro de 2015.

Resumo

O objetivo com este estudo foi analisar a relação entre a conexão visual (sensorial) e a conexão emocional dos consumidores com a intenção de comprar um produto feminino (roupas íntimas) e o efeito moderador do estado civil nessa relação. Para esse fim, um estudo quantitativo foi realizado com uma amostra por conveniência $(n=241)$, no qual os resultados foram analisados por modelagem de equações estruturais. Concluiu-se que a intenção de comprar lingerie para mulheres na amostra obtida ocorre pela conexão visual e emocional com o produto e que a relação entre conexão emocional e intenção de compra é moderada pelo estado civil da entrevistada.

Palavras-chave: Conexão visual. Conexão emocional. Intenção de compra.

\title{
Visual and emotional connection and the intention of buying lingerie for women and marital status moderation
}

\begin{abstract}
The aim with this study was to analyze the relationship between the visual connection and the emotional connection of the consumer who have the intention to purchase a feminine product (underwear) and the moderating effect of marital status in this relationship. For this, we carried out a quantitative survey by convenience sampling $(n=241)$, in which the results were analyzed by Structural Equation Modeling. It can be concluded that the intention of buying lingerie for women, in the sample considered, occurs by visual and emotional connection with the product and that the relationship between the emotional connection and purchase intention is moderated by the interviwee's marital status.
\end{abstract}

Keywords: Visual connection. Emotional conection. Purchase intention.

\section{INTRODUÇÃO}

O entendimento dos fatores que são capazes de influenciar as decisões de compra se reveste de importância ímpar. Na literatura, tanto fatores relacionados às experiências na compra (MEHARBIAN; RUSSEL, 1974; DONOVAN; ROSSITER, 1982; YALCH; SPANGENBERG, 1990; BAKER et al., 2002; HULTEN, 2011) quanto fatores relacionados às emoções (HOLBROOK, 1982; EDELL; BURKE, 1987; FASEUR; GEUENS, 2006; LERNER; KELTNER, 2000; YI; BAUMGARTNER, 2004; KEMP; BUI; CHAPA, 2012) vêm sendo discutidos e investigados há algumas décadas.

Quanto às expectativas do consumidor com a experiência de consumo, destaca-se a expectativa sensorial (sensory-based), na qual os consumidores, baseados 
nas experiências anteriores ou nos atributos que esperam do produto, presumem que ele tenha determinadas características sensoriais. A expectativa hedônica, relacionada às emoções, impele os consumidores a formarem uma ideia preconcebida do quanto gostarão do produto, antes mesmo da experiência de consumo (CARDELLO, 1993).

Os cinco sentidos foram ignorados durante muito tempo nos estudos de marketing. Apenas recentemente constituíram-se um tema de pesquisa acadêmica e empresarial. Os sentidos humanos tornaram-se, então, objeto central do entendimento da intenção de compra e do comportamento de consumo. Nesse cenário, a propaganda parece não ser mais suficiente para a elaboração de ações que conquistem o consumidor. O marketing sensorial não trata mais de consumo de massa ou segmentação de mercado, mas foca na compreensão de como o indivíduo sente e percebe produtos e serviços, e de que forma os sentidos influenciam o consumo (HULTEN; BROWEUS; VAN DIJK, 2009).

A conexão emocional refere-se ao conhecimento de consumidores, produtos e serviços durante a experiência de consumo. Este, segundo Holbrook (1982), pode ser avaliado de acordo com os sentimentos que são gerados "[...] durante a experiência de uso/consumo", podendo ser tanto sentimentos positivos (ex.: alegria, amor) quanto negativos (ex.: medo, nojo).

Considerando-se esse contexto teórico, ressalta-se a importância de se estudar a relação entre estímulos sensoriais e fatores emocionais relacionados à intenção de compra. Escolheu-se o produto lingerie para averiguar as relações propostas em razão do entendimento de que ele possui forte apelo e valor simbólico para as mulheres, o que sugeriu que o apelo visual e emocional poderia explicar a intenção de comprá-lo.

O objetivo com este estudo é analisar a relação entre a conexão visual e a conexão emocional do consumidor com a intenção de compra de um produto de uso feminino (lingerie) e o efeito moderador do estado civil nessa relação. Para isso, realizou-se uma pesquisa quantitativa na qual foram testadas as hipóteses formuladas.

\section{CONEXÃO VISUAL}

Algumas empresas têm buscado explorar as dimensões sensoriais dos consumidores no ambiente de sua loja, como músicas, cores, iluminação e aroma (SOARS, 2009). Desde 1974, quando Mehrabian e Russel sugeriram que os estímulos ambientais proporcionam um comportamento de evitação ou aproximação nos consumidores, essas dimensões são estudadas. Soars (2009) cita um exemplo que retrata bem essa preocupação cada vez mais forte entre os gestores: empresas de café, como Starbukcs e Caffé Nero, desenham suas lojas para incentivar a experiência de consumo de seus clientes, 
pois em uma pesquisa realizada pela Nespresso se identificou que mais de $60 \%$ das experiências dos consumidores de café vêm do ambiente da loja onde ele é comprado.

Dessa forma, empresas dedicam mais atenção aos mecanismos capazes de estimular a atração dos consumidores para seus estabelecimentos (WARD; DAVIES; KOOIJMAN, 2007) e, se possível, estimular não somente sua permanência, mas também converter esse tempo de permanência em vendas. Uma sensação positiva em um ambiente poderá determinar maior tempo de permanência do consumidor dentro da loja (DONOVAN; ROSSITER, 1982) e, consequentemente, o aumento da probabilidade de ocorrerem compras por impulso (YALCH; SPANGENBERG, 1990).

A forma como os estímulos visuais podem influenciar a intenção de compra dos consumidores não é descrita em profundidade nas teorias de comportamento de compra tradicional (CLEMENT, 2007). No entanto, o design do produto pode ajudar as empresas a ganharem vantagem competitiva e estabelecerem um ponto de diferenciação (LANDWEHR; WENTZEL; HERRMANN, 2013).

A manipulação de fatores experienciais é uma tentativa de comunicar determinada mensagem com a intenção de alcançar objetivos específicos, como resposta imediata para determinado comportamento, por exemplo, "ficar”, "navegar” e "comprar” (WARD; DAVIES; KOOIJMAN, 2007). De acordo com Kooijman (2003), as empresas devem procurar transformar a comunicação caracterizada pela intangibilidade em algo tangível, precisando ainda estar atentas em manipular um estado que provoque vínculo emocional positivo com o cliente, uma vez que isso pode proporcionar o desejado aumento no tempo de permanência.

Alguns componentes de estimulação de ambiente têm sido estudados; os estímulos visuais têm tido maior atenção por parte dos pesquisadores (WARD; DAVIES; KOOIJMAN, 2007), especialmente os voltados à influência de cor, textura, formato, layout (TAI; FUNG, 1997; BAKER et al., 2002) e iluminação (ARENI; KIM, 1994).

Essa é uma discussão em curso e que está sendo pesquisada em diferentes frentes, passando desde a análise de como os produtos estão dispostos nos pontos de venda (CLEMENT, 2007) até a importância do design e do valor estético, tanto no âmbito do produto (ECKMAN; WAGNER, 1994; LANDWEHR; WENTZEL; HERRMANN, 2013) quanto no ambiente de loja como um todo (VIEIRA, 2010).

Não é surpresa, portanto, que estudos apontem que os consumidores têm aumentado sua escolha por marcas tomando por base critérios de valor estético e de distinção de design visual. Um produto com design estético superior, que consiga atrair atenção do consumidor, pode ganhar sua preferência em detrimento dos concorrentes (VIEIRA, 2010). 
O estudo da estética, apesar de estar associado a todo o tipo de arte, é usualmente associado às artes visuais. Ele pode, no entanto, ser estendido para o design de objetos do cotidiano, para sua aparência, para o que é consumido visualmente (ECKMAN; WAGNER, 1994; VIEIRA, 2010). O design é compreendido como a combinação única de elementos visuais - linha, espaço, forma, luz, cor e padrão (ECKMAN; WAGNER, 1994).

Um bom design “[...] atrai os consumidores a um produto, se comunica com eles, e agrega valor ao produto, aumentando a qualidade das experiências de uso associados.” (BLOCH, 1995, p. 16). Muito tem-se discutido sobre como os elementos do design estão relacionados uns aos outros para determinar a atratividade do objeto. Historicamente, o design do produto tem sido considerado um processo para criar diferenciação funcional por meio de recursos adicionais, desempenho superior, entre outros elementos. No entanto, cada vez mais o design estático do produto passa a ser visto como um “[...] elemento estratégico importante na criação de preferência e valor emocional mais profundo para o consumidor”, permitindo a criação de elementos de conexão visual (NOBLE; KUMAR, 2008, p. 441).

O julgamento do consumidor acerca desses elementos é afetado por atributos visuais e configurações de atributos visuais (ECKMAN; WAGNER, 1994). Os estímulos e as características visuais podem exercer impacto na estratégia de pesquisa adotada pelo consumidor e no seu tempo de resposta, durante a tomada de decisão de compra (CLEMENT, 2007). Noble e Kumar (2008) demonstram que os diferentes elementos de design podem ser usados de forma estratégica para criar duas cadeias de resultados muito diferentes do ponto de vista do consumidor. Alguns elementos de design são mais propensos a criar diferenciação do produto funcional e resultados para os consumidores transacionais, enquanto outras estratégias de design podem tocar de forma mais emocional na criação de valor para o consumidor. Os elementos de foco emocional na criação de valor são mais suscetíveis de gerar resultados como lealdade, alegria de uso e até mesmo paixão.

Para avaliar o apelo relativo de produtos que diferem na tipicidade (designs mais arrojados ou produtos considerados mais tradicionais), Landwehr, Wentzel e Herrmann (2013) sugerem que deve ser analisado em que medida os consumidores estão realmente expostos aos produtos, antes da tomada de decisão. Os autores referem que o tempo de exposição do consumidor aos produtos permite melhor compreender e prever o sucesso de diferentes produtos no mercado, uma vez que, estando em maior contato com algo de design mais arrojado, o consumidor tende a valorizá-lo diante daquele tradicional, demonstrando intenção de compra. 
A diferenciação funcional, objetivo mais comum do design, leva a resultados transacionais, como escolha e satisfação das expectativas. Embora importante para as vendas de curto prazo das empresas, esses resultados não provocam, necessariamente, benefícios de longo prazo. Contudo, se alcançado o êxito na criação de valor emocional, as empresas são mais propensas a gerar resultados relacionais de longo prazo, como lealdade, compromisso e, até mesmo, paixão pelo produto. Esses resultados indicam vida mais longa da relação com o cliente, além de benefícios auxiliares, como a geração de boca a boca positivo (NOBLE; KUMAR, 2008).

O aparecimento de um novo produto também influencia a percepção dos consumidores quanto a funcionalidades, qualidade e facilidade de utilização. Isso sugerem Creusen, Veryzer, Schoormans (2010), quando avaliam como a preferência para a complexidade visual e a simetria dependem do valor que o produto tem para o consumidor, ou seja, o que chama sua atenção. Nesse sentido, trabalhos importantes já foram realizados na busca da compreensão das reações cognitivas e emocionais dos consumidores para design de produto e aparência (BLOCH, 1995; CREUSEN; SCHOORMANS, 2005; CRILLY; MOULTRIE; CLARKSON, 2004).

Bloch (1995) apresenta um modelo de respostas do consumidor à forma do produto, no qual a indica como estando associada à sua performance, ergonomia, produtividade/custo, design, programa de marketing e regulação legal, e sendo influenciada por elementos moderadores, como os gostos pessoais e as preferências (afetados por fatores culturais e de contexto e por características como experiência, personalidade), além de fatores situacionais. Esses elementos, diz o autor, influenciam nas respostas psicológicas (cognitivas e afetivas) em relação ao produto. Essas respostas é que farão o consumidor dele se aproximar (intenção de compra) ou evitá-lo.

Para Creusen e Schoormans (2005), a interpretação simbólica é parte da experiência estética, sendo o valor estético indicado tanto como impressão hedônica quanto como resultado de interpretação e representação (SCHMITT; SIMONSON, 1997). Dadas as tendências de gestão de negócios baseadas em relacionamento, a ênfase na criação de conexão emocional e visual, gerando valor emocional por meio de produto, é particularmente relevante (NOBLE; KUMAR, 2008).

O valor simbólico é considerado uma razão para a escolha de um produto, decorrente de várias associações - barato, caro, amigável, profissional - feitas pelos consumidores. Os objetos também podem retratar tendências (por exemplo, objeto moderno ou contemporâneo). Valores estéticos e simbólicos, muitas vezes, estão juntos na percepção do consumidor e podem entrar em conflito quando da decisão de compra. Uma pessoa pode gostar da aparência de certo objeto, mas não comprá-lo, 
porque as associações simbólicas não são adequadas à sua pessoa (por exemplo, algo com aparência infantil para um adulto) ou à ocasião (para uso em casa ou em uma festa) (CREUSEN; SCHOORMANS, 2005).

\subsection{CONEXÃO EMOCIONAL}

As emoções desempenham importante papel na formação de atitudes e julgamentos por parte dos consumidores (EDELL; BURKE, 1987; FASEUR; GEUENS, 2006). Lerner e Keltner (2000) afirmam que diferentes emoções - como a simpatia, a tristeza, o orgulho, a raiva - têm resultado significativo sobre as escolhas e a tomada de decisão de um indivíduo. Como os consumidores lidam com as emoções é uma questão importante em relação ao marketing, uma vez que os sentimentos podem influenciar comportamentos pós-compra, como recompra, boca a boca positivo e negativo (YI; BAUMGARTNER, 2004).

Durante as últimas décadas, muitas organizações direcionaram seus esforços em pesquisas para entender as emoções (KEMP; BUI; CHAPA, 2012). Associam-se essas considerações às de Palmer e Koenig-Lewis (2010), para os quais as emoções têm emergido como um componente cada vez mais importante de satisfação do cliente e como antecedente de recompra e de comportamento de referência. O consumo de produtos ou serviços pode ajudar a desviar a atenção de sentimentos indesejados e permitir a reorientação a estados desejados, destacam Kacen e Lee (2002).

Palmer e Koenig-Lewis (2010) afirmam que, em meio à complexidade de definir e medir as emoções, não está claro se as emoções positivas e negativas têm efeitos sobre satisfação e comportamento de recompra. Os autores destacam que é possível, por exemplo, que as emoções negativas afetem comportamentos como a satisfação e a intenção de recompra, enquanto as emoções positivas afetam outros componentes.

Muitas pesquisas têm demonstrado que as emoções (LERNER; KELTNER, 2000; LOEWENSTEIN; DONOGHUE, 2004)contrasting the effects of positive versus negative feeling states. These approaches have not specified if and when distinct emotions of the same valence have different effects on judgement. In this article, we propose a model of emotion-specific influences on judgement and choice. We posit that each emotion is defined by a tendency to perceive new events and objects in ways that are consistent with the original cognitive-appraisal dimensions of the emotion. To pit the valence and appraisal-tendency approaches against one another, we present a study that addresses whether two emotions of the same valence but differing appraisalslu2014anger and fearlu2014relate in different ways to risk perception. Consistent 
with the appraisal-tendency hypothesis, fearful people made pessimistic judgements of future events whereas angry people made optimistic judgements. In the Discussion we expand the proposed model and review evidence supporting two social moderators of appraisal-tendency processes.”, “author” : [ \{ “dropping-particle” : “”, "family” : “Lerner”, “given” : “Jennifer S.”, “non-dropping-particle” : “”, “parse-names” : false, “suffix” : “” \}, \{ “dropping-particle” : “”, “family” : “Keltner”, “given” : “Dacher”, “non-dropping-particle” : “”, “parse-names” : false, “suffix” : “” \} ], “container-title” : “Cognition \& Emotion”, “id” : “ITEM-1”, “issued” : \{ “date-parts” : [ [ “2000” ] ] \}, “page” : “473-493”, “title” : “Beyond valence: Toward a model of emotion-specific influences on judgement and choice”, “type” : “article”, “volume” : “14” \}, "uris” : [ "http://www.mendeley.com/documents/?uuid=d5b213f2-429d-4eb7-9db7-33ef3b3f15d7” ] \}, \{ “id” : “ITEM-2”, “itemData” : \{ “DOI” : “10.2139/ssrn.539843”, “ISSN” : “1556-5068”, “abstract” : “This article considers a market served by a monopolist who sells a durable good that depreciates stochastically over time. We show that there exist three types of stationary equilibria: a Coase Conjecture equilibrium, a monopoly equilibrium, and a reputational equilibrium. When the depreciation rate is low, the Coase Conjecture equilibrium is the unique equilibrium. For intermediate values of the depreciation rate, all three equilibrium types coexist. When the depreciation rate is high, the monopoly equilibrium is the unique equilibrium. Consequently, when selling a good of sufficiently low durability, the monopolist does not lose any of her monopoly power. Furthermore, the steady-state output in the reputational equilibrium falls below the monopoly quantity. Hence, in durable goods markets, welfare losses due to monopoly power may be larger than in markets for perishables. Copyright (c, os humores (KIM; PARK; SCHWARZ, 2010), o afeto (HSEE; ROTTENSTREICH, 2004) e, até mesmo, o clima (SCHWARZ; CLORE, 1983) podem ter efeito nos julgamentos e nas avaliações das pessoas sobre produtos e experiências. Alguns autores examinaram como as emoções positivas ou negativas, em geral, podem influenciar o julgamento e a tomada de decisão dos consumidores (GORN; GOLDBERG; BASU, 1993). Griskevicius; Shiota e Nowlis (2010) constataram que o orgulho eleva a intenção de compra de determinados produtos (ex.: relógios) e que o fator “contentamento” eleva a intenção de compra de produtos para casa (ex.: máquinas de lavar louça).

Outro exemplo de como as emoções podem induzir determinados comportamentos é apresentado por Winkielman e Berridge (2004). Eles explicam que as bebidas podem reduzir estímulos relacionados às emoções negativas. Ellsworth e Smith (1988) relatam que, ao ler um menu de restaurante, muitos consumidores não estão 
cientes dos sentimentos sutis de excitação implícitos e induzidos por apresentações visualmente atraentes de escolhas alimentares pouco saudáveis.

A literatura sobre o comportamento do consumidor sugere que os indivíduos podem se envolver emocionalmente com certos aspectos durante o processo de consumo (MICK; DEMOSS, 1990; ANDRADE, 2005). Por exemplo: a compra de um produto que oferece algum benefício prazeroso (ex.: joias, carros, etc.), ou a prática de algum comportamento moderadamente “indulgente” (ex.: comer chocolate) podem ajudar as pessoas a regularem determinados estados afetivos negativos.

Utilizando informações sobre a teoria do “afeto”, Schwarz e Clore (1983) descobriram que as pessoas usam suas emoções positivas ou negativas como base para julgamentos de produtos, anúncios e extensões de marca. Nesse sentido, Berthon, Ewing e Hah (2005), Faseur e Geuens (2006) Micu e Chowdhury (2010) e Shimp e Stuart (2004) demonstraram que a publicidade tem a capacidade de provocar respostas emocionais no consumidor.

Em outra pesquisa, Laran e Wilcox (2011) verificaram que os efeitos de emoções positivas específicas podem variar em decorrência dos fatores associados à experiência de emoções positivas específicas; por exemplo, o orgulho pode aumentar ou diminuir o comportamento indulgente dos consumidores.

Muitos estudos têm confirmado a experiência familiar e os efeitos do humor sobre o julgamento avaliativo, incluindo a avaliação de produtos de consumo (COHEN; PHAM; ANDRADE, 2008; SCHWARZ; CLORE, 2007; TUAN PHAM, 2004)1991. O tema emoções tem atraído considerável interesse no campo da pesquisa de satisfação do cliente.

Apesar da contribuição significativa para a compreensão do papel das emoções no marketing, a maioria dos trabalhos tem sido transversal e, por consequência, não capta plenamente a natureza das experiências dos consumidores ao longo do tempo (BOLTON; LEMON, 1999).

\subsection{HIPÓTESES}

Perante esse contexto teórico, foram elaboradas as hipóteses do presente estudo: entendeu-se que a intenção de compra de um produto poderia ser predita por elementos relacionados à experiência de consumo (fatores sensoriais) e ao apelo emocional advindo do produto. Nesse trabalho, utilizou-se o produto lingerie pelo entendimento de tratar-se de um produto cujas características provocariam, nas consumidoras, ligação emocional (hedônica) e forte apelo visual decorrentes das cores e 
dos detalhes de cada peça. Entendeu-se, também, nesse contexto, que o estado civil poderia moderar as relações hipotetizadas.

H1- A conexão visual prediz a intenção de compra de lingerie.

H2- A conexão emocional prediz a intenção de compra de lingerie.

H3: O estado civil modera a relação entre a conexão visual e emocional e a intenção de compra.

Esquema 1 - Modelo teórico

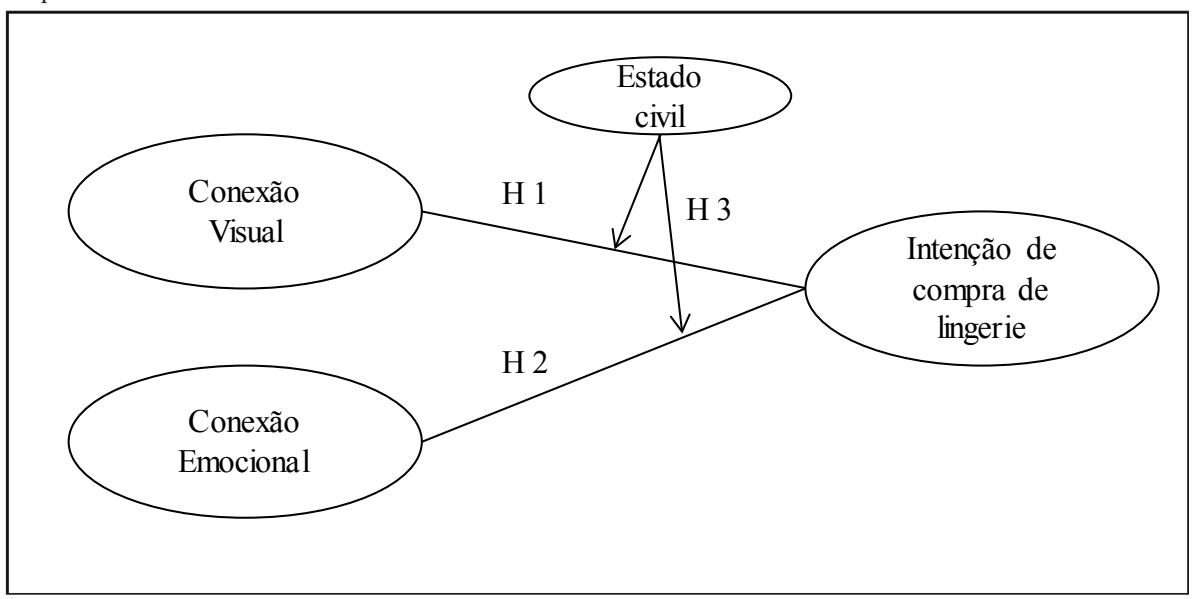

Fonte: os autores.

\section{PROCEDIMENTOS METODOLÓGICOS}

Com o intuito de analisar as hipóteses propostas no modelo de pesquisa, foi utilizada uma pesquisa descritiva com abordagem quantitativa (HAIR JÚNIOR et al., 2009), tendo como foco a elaboração de um projeto formal, visando à obtenção de dados de forma empírica, sistemática e objetiva para o alcance do objetivo proposto. Neste trabalho, optou-se pela coleta de dados por intermédio de questionários estruturados, desenvolvidos a partir da teoria revisada, e pela amostragem por conveniência.

O instrumento de coleta foi construído a partir das teorias revisadas para os construtos conexão visual e conexão emocional. O construto intenção de compra foi oriundo da escala de Bruner e Hensel (1998). O instrumento original era formado por 23 questões: 8 itens referentes à conexão visual; 8 itens referentes à conexão emocional; 5 itens referentes à intenção de compra. $\mathrm{O}$ instrumento original passou por um pré-teste com 38 respondentes e foi analisado com utilização da análise fatorial exploratória, a qual reduziu a quantidade de itens, de acordo com suas cargas fatoriais 
e comunalidades. Seguindo recomendação de Hair Júnior et al. (2009), todos os itens com cargas abaixo de 0,5 foram excluídos.

O instrumento foi, então, aplicado, presencialmente, a 253 respondentes. Após a análise inicial, foram excluídos questionários não totalmente respondidos, rasurado, que dicotomizaram a escala e aqueles dos quais todas as assertivas foram respondidas na mesma opção, restando 241 questionários válidos para a análise.

Quanto aos dados socioeconômicos, a amostra foi composta apenas por mulheres residentes na Cidade de Porto Alegre, predominantemente com renda individual entre R\$ 1.000,00 e R \$ 3.000,00, ensino médio completo e ensino superior, idades entre 19 e 45 anos conforme a Tabela 1.

Tabela 1 - Composição da amostra

\begin{tabular}{llllllll}
\hline $\mathrm{n}$ & Renda mensal & n. & Escolaridade & n. & Idade & n. & Estado Civil \\
\hline 52 & até R\$ 1.000 & 2 & Ensino fundamental & 14 & menos de 18 anos & 123 & Solteira \\
\hline 144 & de R\$ 1.001a R\$ 3.000 & 97 & Ensino médio & 39 & de 19 a 25 anos & 65 & Casada \\
\hline 41 & de R\$ 3.001a R 5.000 & 112 & Ensino superior & 92 & de 26 a 35 anos & 24 & União estável \\
\hline 03 & de R\$ 5.001 a 10.000 & 28 & Pós-graduado & 63 & de 36 a 45 anos & 24 & Divorciada \\
\hline 0 & acima de 10.001 & 1 & Mestre & 30 & de 45 a 60 anos & 3 & Viúva \\
\hline 01 & outros & 1 & Doutor & 03 & acima de 61 anos & 2 & Outros \\
\hline
\end{tabular}

Fonte: os autores.

O procedimento de análise adotado foi a Modelagem de Equações Estruturais (MEE). Dentro do procedimento de MEE, também se utilizou a análise multigrupos para avaliar a moderação do fator estado civil. Essa técnica examina uma série de relações por meio de um conjunto de métodos que identifica variáveis latentes, utilizando um conjunto de técnicas multivariadas e examinando múltiplas relações de dependência simultaneamente entre essas variáveis (SCHUMACKER; LOMAX, 1996; HAIR JÚNIOR et al., 2009).

Para os ajustes finais do modelo e de suas hipóteses, foram utilizados os seguintes índices: Qui-quadrado, graus de liberdade, nível de probabilidade, Comparative Fit Index (CFI), Normed Fit Index (NFI), Root Mean Squared Error of Approximation (RMSEA), Variância Extraída, confiabilidade Alfa de Crombach. Os critérios para avaliação da adequação do modelo são os sugeridos por Mulaik et al. (1989), Byrne (1998), Hair Júnior et al. (2009) e Arbuckle (2008). Utilizou-se para avaliar os resultados coletados Modelagem de Equações Estruturais para testar o modelo hipotético. As escalas foram testadas em dimensionalidade, confiabilidade e validade, utilizando análise fatorial confirmatória (AFC). 


\section{RESULTADOS}

A primeira reflexão realizada, antes da análise das hipóteses levantadas, foi a verificação dos pressupostos de normalidade das variáveis observadas. Para isso, aplicou-se a análise fatorial confirmatória. Ela serviu, no primeiro momento, para calcular os indicadores de curtose e assimetria. O coeficiente de Mardia foi realizado para análise da curtose de cada variável observável. Os resultados demonstraram normalidade nas distribuições dentro dos pressupostos pautados por Bentler (1995) e Mardia (1971). Os dados apresentaram assimetria moderada, já que os coeficientes de assimetria de Pearson foram próximos a zero.

Posteriormente, a etapa consistiu na verificação da qualidade dos dados, o que ocorreu pela análise dos outliers univariados e multivariados, além da avaliação dos casos omissos. A verificação dos outliers univariados se desenvolveu por meio da análise dos escores Z. Nesses casos, foram excluídos da análise final aqueles casos que apresentaram valores superiores a $|3|$ para cada variável (HAIR JÚNIOR et al., 2009). Nessa etapa identificaram-se 15 casos dentro dessa situação, os quais foram excluídos da amostra final. A etapa para verificação dos outliers multivariados foi efetivada por meio da análise de Mahalanobis. Nesse caso, verificou-se se houve distância grande entre o valor individual e as médias amostrais obtidas (KLINE, 1998; JÖRESKOG, 1993). Nessa etapa, excluíram-se mais dois casos da análise final.

A última etapa da análise preliminar consistiu na avaliação dos casos omissos, e também se realizou a análise de frequência de todas as questões do instrumento de pesquisa. Foi observado se havia alguma questão no instrumento que pudesse ter sido mal interpretada ou não entendida. Se isso ocorresse, seria interessante excluir a questão que tivesse obtido mais de 10\% de não resposta para a variável; contudo, tal situação não foi identificada.

Na etapa seguinte, avaliou-se individualmente cada questionário respondido, para verificar se existia algum com índice de não resposta superior a 10\% no instrumento final. Nessa etapa identificaram-se 15 questionários com esse problema, os quais foram retirados da amostra final.

\subsection{CONFIABILIDADE DO INSTRUMENTO}

A etapa para verificação da confiabilidade do instrumento de pesquisa se desenvolveu pela avaliação de Alpha de Cronbach, confiabilidade composta e validade discriminante e convergente dos construtos utilizados. Na primeira análise, Alpha de 
Cronbach, visou-se avaliar a consistência interna das escalas utilizadas (confiabilidade simples), cujo índice tolerável deve ser superior a 0,6 (HAIR JÚNIOR et al., 2009). Todos os construtos apresentaram índices aceitáveis - intenção de compra $(0,894)$, conexão visual $(0,868)$ e conexão emocional $(0,870)$. Em relação à confiabilidade composta, os índices apresentados foram adequados: intenção de compra, 0,90, conexão visual, 0,90, e conexão emocional, 0,91.

Finalizando, checou-se a validade discriminante e convergente dos construtos. Salienta-se que as cargas fatoriais dos itens sobre cada variável latente evidenciaram loadings significativos a 0,01. A Tabela 2 apresenta a raiz de correlação, na qual é apresentada a variância extraída de cada construto (AVE), assim como a variância compartilhada (VC). Os resultados demonstram que as variáveis extraídas são sempre menores que as confiabilidades compostas dos construtos, confirmando, dessa forma, a validade discriminante e convergente.

Tabela 2 - Validade Convergente e Discriminante

\begin{tabular}{lrrr}
\hline Construtos & Conexão Visual & Conexão Emocional & Intenção de compra \\
\hline Conexão visual & $0,79 *$ & & \\
Conexão Emocional & $0,69^{* *}$ & $0,89^{*}$ & \\
Intenção de compra & $0,53^{* *}$ & $0,62^{* *}$ & $0,86^{*}$ \\
\hline
\end{tabular}

Fonte: os autores.

Nota: * Confiabilidade Composta ** Variância Extraída

\subsection{ANÁLISE DO MODELO INTEGRADO}

A análise do modelo integrado, que agrega o modelo de mensuração e o modelo estrutural, partiu das três dimensões consideradas no estudo. A avaliação do referido modelo teve como base a reflexão dos índices de ajustamento e da significância estatística e dos coeficientes de regressão estimados (KLINE, 1998; MARUYAMA, 1998). Os índices também foram norteadores para analisar as primeiras hipóteses da pesquisa.

No modelo final, adaptações foram realizadas conforme sugestões propostas no software Amos 18.0. Essa iniciativa se justifica para a melhor explicação e o melhor entendimento do fenômeno estudado, pois, no modelo original (sem as correlações propostas pelo Amos), os índices apresentados ficaram abaixo do desejável, uma vez que as medidas de NFI, IFI, TLI e CFI ficaram bem abaixo do desejável $(0,9)$, o índice de RMSEA. As correlações sugeridas somente seriam aceitas se houvesse alguma argumentação lógica/teórica para elas. Foi realizada a correlação entre os 
construtos conexão visual e conexão emocional. A Tabela 3 apresenta os coeficientes padronizados e a significância do modelo integrado.

Tabela 3 - Teste das hipóteses

\begin{tabular}{|c|c|c|c|c|c|c|}
\hline Variável independente & & $\begin{array}{l}\text { Variável depen- } \\
\text { dente }\end{array}$ & $\begin{array}{l}\text { Coeficiente padro- } \\
\text { nizado }\end{array}$ & Desvio padrão & $\mathrm{Z}$ & $\mathrm{P}$ \\
\hline Conexão visual & $\leftarrow$ & $\begin{array}{l}\text { Intenção de } \\
\text { compra }\end{array}$ & 0,216 & 0,109 & 2,166 & $*$ \\
\hline Conexão emocional & $\leftarrow$ & $\begin{array}{l}\text { Intenção de } \\
\text { compra }\end{array}$ & 0,466 & 0,122 & 4,387 & $* *$ \\
\hline
\end{tabular}

Fonte: os autores.

Nota: $(* *)<0,001 ;(*)<0,05$

Observa-se, pelos resultados apresentados na Tabela 2, a confirmação das relações hipotetizadas, uma vez que houve impactos positivos dos construtos design hedônico e conexão sensorial na intenção de compra de lingerie, por parte das mulheres respondentes. A seguir são demonstrados os índices de ajustamento do modelo integrado, original e com ajustes (Tabela 4).

Tabela 4 - Índice de ajuste do modelo 1

\begin{tabular}{lll}
\hline Índice & Análise & \\
\hline Qui-quadrado & Inicial & Final \\
\hline Graus de liberdade & 415,802 & 268,814 \\
\hline Qui-quadrado/graus de liberdade & 88 & 82 \\
\hline CFI - Comparative Fit Index & 4,72 & 3,27 \\
\hline NFI - Normed Fit índex & 0,823 & 0,899 \\
\hline IFI & 0,789 & 0,864 \\
\hline TLI & 0,826 & 0,901 \\
\hline RMSEA & 0,759 & 0,853 \\
\hline
\end{tabular}

Fonte: os autores.

Ao se refletir sobre os índices apresentados no modelo inicial e final (com os ajustes realizados, conforme descrito anteriormente), observa-se considerável evolução, pois houve melhor relação entre “qui-quadrado/graus de liberdade”, aproximação dos índices adequados de CFI, NFI, TLI e RMSEA, além do alcance do índice adequado de IFI (MULAIK et al., 1989; BLUNCH, 2008).

\subsection{ANÁLISE DO EFEITO MODERADOR ESTADO CIVIL}


Esta etapa visou avaliar se o efeito moderador do estado civil das respondentes poderia impactar a relação entre a conexão visual e a conexão emocional em relação à intenção de compra. Para isso, aplicou-se a técnica de modelagem de equações estruturais multigrupos, dividindo a amostra em dois grupos: um composto pelas entrevistadas que responderam ser solteiras, e outro, por mulheres que possuem relação estável, ou seja, que estão namorando ou são casadas. Na análise de multigrupos, um modelo tem todos os caminhos fixos, exceto o caminho que se quer testar como diferente entre os grupos (BYRNE, 2009). Apresenta-se, na Tabela 5, a análise do efeito moderador entre os grupos analisados.

Tabela 5 - Análise do efeito moderador

\begin{tabular}{llll}
\hline Trajetórias & Solteiras & Casadas & $\begin{array}{l}\text { Diferença } \\
\mathrm{X}^{2}\end{array}$ \\
\hline Conexão Visual $\leftarrow$ Intenção de compra & $-0,186$ & $0,409 *$ & 0,804 \\
\hline Conexão emocional $\leftarrow$ Intenção de compra & $0,922^{* *}$ & 0,230 & 0,010 \\
\hline
\end{tabular}

Fonte: os autores.

Nota: $(* *)<0,001 ;(*)<0,05$.

Os resultados demonstram a existência do efeito moderador do estado civil na relação entre conexão emocional e intenção de compra, enquanto na relação conexão visual e intenção de compra não se observa tal comportamento, pois o índice de $\mathrm{X}^{2}$ ficou acima de 0,05 , não sustentando diferença estatística dos comportamentos nos modelos distintos analisados.

\subsection{REFLEXÕES SOBRE AS HIPÓTESES}

Ao se aprofundar a relação em que houve comprovação do efeito moderador do estado civil, detecta-se que a relação conexão emocional e intenção de compra foi mais forte para as mulheres solteiras. Para as casadas, a relação foi fraca e não significativa. Tais resultados comprovam as duas primeiras hipóteses e a reconsideração da terceira. O Esquema 2 apresenta o modelo resultante. A seguir, é evidenciada a reflexão sobre as hipóteses. 


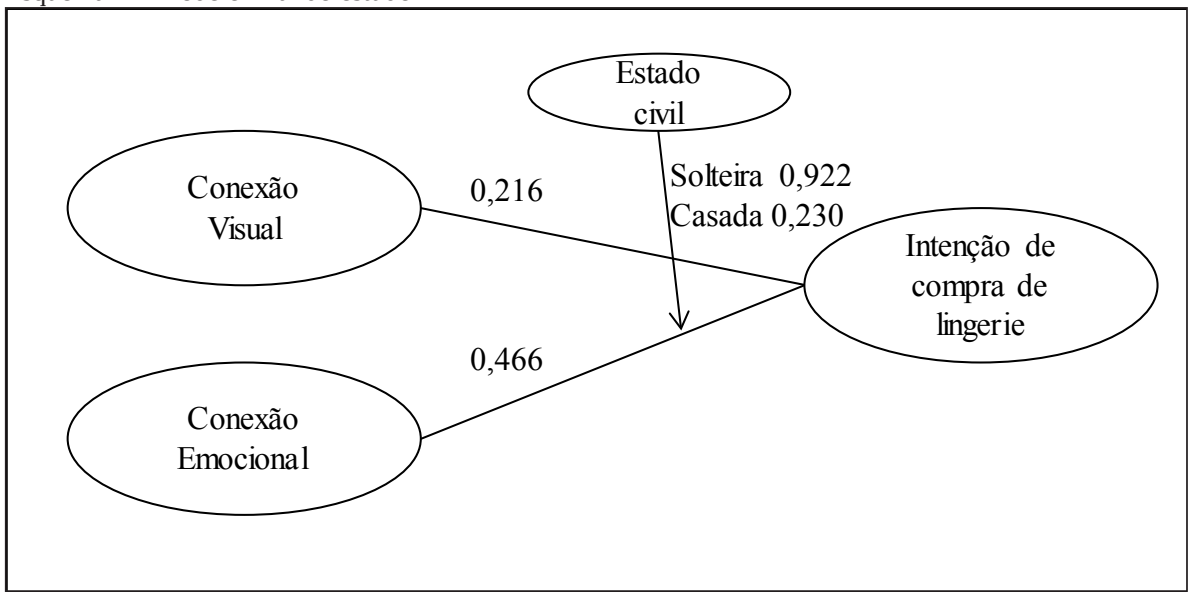

Fonte: os autores.

As hipóteses testadas H1 (a conexão visual prediz a intenção de compra de lingerie) e H2 (a conexão emocional prediz a intenção de compra de lingerie) foram confirmadas. Embora as relações testadas não sejam propriamente fortes, são relações existentes.

Quanto à H1, a literatura sugere que os elementos visuais podem anteceder a intenção de compra, conforme explicitado em Vieira (2010), Bloch (1995), Eckman e Wagner (1994), o que comprova a predição encontrada nas teorias consultadas. Referente à H2, encontra-se, na literatura, o forte papel das emoções nas decisões de compras (EDELL; BURKE, 1987; LERNER; KELTNER, 2000; LOEWENSTEIN; DONOGHUE, 2004; FASEUR; GEUENS, 2006), as quais podem influenciar também os comportamentos pós-compra (YI; BAUMGARTNER, 2004). Os indivíduos, durante o processo de consumo, podem se envolver emocionalmente com certos aspectos (MICK; DEMOSS, 1990; ANDRADE, 2005). A hipótese H1 reflete o que já havia sido observado na teoria. Pode-se entender que a intenção de compra de lingerie é predita pela conexão visual.

A H3 (o estado civil modera a relação entre a conexão visual e emocional e a intenção de compra) precisou, no entanto, ser revisada, pois o efeito moderador aconteceu apenas em mulheres solteiras na conexão emocional. A revisada foi reescrita da seguinte forma: o estado civil modera a relação entre a conexão emocional e a intenção de compra em mulheres solteiras. 


\section{CONCLUSÃO}

O presente estudo analisou a relação da conexão visual e emocional com a intenção de compra e a moderação do estado civil. No primeiro momento, os resultados confirmaram as trajetórias descritas nas duas primeiras hipóteses, que prediziam a relação da conexão visual e da conexão emocional com a intenção de compra de lingerie. Essa descoberta fornece algumas evidências adicionais para os estudos em questões sensoriais e emoções no consumo de produtos. No segundo momento, a hipótese moderadora demonstrou que dependendo do estado civil da respondente se altera a relação entre a conexão emocional e a intenção de compra de lingerie.

Do ponto de vista gerencial, identificam-se, com essa análise, dois fatores que podem ser ressaltados pelas empresas para aumentar a intenção de compra do produto em foco. Na perspectiva acadêmica, este estudo contribui com uma análise inicial que pode gerar uma escala ligada a consumo de produtos semelhantes ao analisado neste trabalho.

Ressalta-se que limitações importantes, como a amostra e a própria escolha de um único produto para validar as relações propostas, não permitem a generalização dos resultados. A amostra destacada neste estudo restringe os resultados à própria amostra, por não ter sido distribuída proporcionalmente e por ter sido aplicada por conveniência. A escolha de um produto restringe os resultados a ele mesmo. A testagem em outros produtos e populações aumentaria a chance de generalização. As motivações para os resultados encontrados não foram investigadas a fundo. Uma pesquisa qualitativa poderia ampliar a reflexão sobre o modelo.

\section{REFERÊNCIAS}

ANDRADE, E. B. Behavioral Consequences of Affect: Combining Evaluative and Regulatory Mechanisms. Journal of Consumer Research, v. 32, i. 3, p. 355-362, 2005.

ARBUCKLE, J. L. Amos 17 user's guide. Chicago: SPSS, 2008.

ARENI, C; KIM, D. The influence of in-store lighting on consumers examination of merchandise in a wine store. International Journal of Research in Marketing, v. 11, i. 2, p. 117-125, 1994. 
BAKER, J. et al. The influence of multiple store environment cues on perceived merchandise values and patronage intentions. Journal of Marketing, v. 66, p. 120141, 2002.

BARSKY, J.; NASH, L. Evoking Emotion: Affective Keys to Hotel Loyalty The Cornell Hotel and Restaurant. Administration Quarterly, v. 43, i. 1, p. 47-59, 2002.

BENTLER, P. M. Comparative fit indexes in structural equations. Psychological Bulletin, v. 107, i. 2, p. 238-246, 1995.

BERTHON, P.; EWING, M.; HAH, L. L. Captivating company: dimensions of attractiveness in employer branding. International Journal of Advertising, v. 24, p. 151-217, 2005.

BLOCH, P. H. Seeking the ideal form: product design and consumer response. Journal of Marketing, v. 59, i. 3, p. 16-29, 1995.

BLUNCH, N. J. Introduction to structural equation modelling using SPSS and AMOS. London: SAGE Publications, 2008.

BOLTON, R. N.; LEMON, K. N. A Dynamic Model of Customers’ Usage of Services: Usage as an Antecedent and Consequence of Satisfaction. Journal of Marketing Research, v. 36, p. 171-186, 1999.

BRUNER II, G. C.; HENSEL, P. J. Marketing Scales Handbook: a compilation of multi-intens measures. Chicago: American Marketing Association, 1998. v. 2.

BYRNE, B. M. Structural Equation Modeling: Basic Concepts, Application, and Programming. Mahwah, NJ: Lawrence Erlbaum Associated, 1998.

CARDELLO, A. V. What do consumers expect from low-cal, low-fat, lite foods? Cereal Foods Word, v. 38, i. 2, p. 96-99, 1993.

CLEMENT, J. Visual influence on in-store buying decisions: an eye-track experiment on the visual influence of packaging design. Journal of Marketing Management, v. 23, i. 9-10, p. 917-928, 2007.

COHEN, J. B.; PHAM, M. T.; ANDRADE, E. B. The nature and role of affect in consumer behavior. Handbook of consumer psychology, p. 297-348, 2008. 
CREUSEN, M. E. H.; SCHOORMANS, J. P. L. The different roles of product appearance in consumer choice. Journal of Product Innovation Management, v. 22, i. 1, p. 63-81, 2005.

CREUSEN, M. E. H.; VERYZER, R. W.; SCHOORMANS, J. P. L. Product value importance and consumer preference for visual complexity and symmetry. European Journal of Marketing, v. 49, i. 9-10, p. 1437-1452, 2010.

CRILLY, N.; MOULTRIE, J.; CLARKSON, P. J. Seeing things: consumer response to the visual domain in product design. Design Studies, v. 25, p. 547-577, 2004.

DONOVAN, R. J.; ROSSITER, J. R. Store atmosphere: an environmental psychology approach. Journal of Retail, v. 58, p. 34-57, 1982.

ECKMAN, M.; WAGNER, J. The attractiveness of product design: the effect of visual attributes and consumer characteristics. Advances in Consumer Research, v. 21, p. 560-564, 1994.

EDELL, J. A.; BURKE, M. C. The power of feelings in understanding advertising effects. Journal of Consumer Research, v. 14, p. 421-433, 1987.

ELLSWORTH, P. C.; SMITH, C. A. From appraisal to emotion: Differences among unpleasant feelings. Motivation and Emotion, v. 12, i. 3, p. 271-302, 1988.

FASEUR, T.; GEUENS, M. Different positive feelings leading to different ad evaluations: the case of coziness, excitement, and romance. Journal of Advertising, v. 35 , i. 4, p. 129-139, 2006.

GORN, G. J.; GOLDBERG, M. E.; BASU, K. Mood, Awareness, and Product Evaluation. Journal of Consumer Psychology, v. 2, p. 237-256, 1993.

GRISKEVICIUS, V.; SHIOTA, M. N.; NOWLIS, S. M. The many shades of rosecolored glasses: an evolutionary approach to the influence of different positive emotions. Journal of Consumer Research, v. 37, i. 2, p. 238-250, 2010.

HAIR JÚNIOR, J. F. et al. Análise multivariada de dados. 6. ed. Porto Alegre: Artmed, 2009.

HOLBROOK, M. B. The varieties of consumption experience: comparing two typologies of emotion in consumer behavior. Journal of Consumer Research, v. 13, 1982. 
HSEE, C. K.; ROTTENSTREICH, Y. Music, pandas, and muggers: on the affective psychology of value. Journal of Experimental Psychology, v. 1, p. 23-30, 2004.

HULTEN, B. Sensory marketing: the multi-sensory brand-experience concept. European Business Review, v. 23, p. 256-73, 2011.

HULTEN, B.; BROWEUS, N.; VAN DIJK, M. Sensory Marketing. Palgrave Macmillan: Basingstoke, 2009.

JÖRESKOG, K. G. Testing structural equation models. Thousand Oaks: Sage, 1993.

KACEN, J. J.; LEE, J. A. The influence of culture on consumer impulsive buying behavior. Journal of Consumer Psychology, v. 12, p. 163-176, 2002.

KEMP, E.; BUI, M.; CHAPA, S. The role of advertising in consumer emotion management. International Journal of Advertising, v. 31, i. 2, p. 339, 2012.

KIM, H.; PARK, K.; SCHWARZ, N. Will this trip really be exciting? The role of incidental emotions in product evaluation. Journal of Consumer Research, v. 36, i. 6, p. 983-991, 2010.

KLINE, R. Principles and practice in structural equation modeling. New York: Guilford Press, 1998.

KOOIJMAN, D. Shop-image and interaction: the use of senses in consumption space. Design and Emotion, London: Taylor \& Francis, 2003.

LANDWEHR, J. R.; WENTZEL, D.; HERRMANN, A. Product design for the long run: consumer responses to typical and atypical designs at different stages of exposure. Journal of Marketing, v. 77, p. 92-107, 2013.

LARAN, J.; WILCOX, K. Choice, Rejection, and Elaboration on Preference-Inconsistent Alternatives. Journal of Consumer Research, v. 38, p. 229-241, 2011.

LERNER, J. S.; KELTNER, D. Beyond valence: Toward a model of emotion-specific influences on judgment and choice. Cognition \& Emotion, v. 14, p. 473-493, 2000. 
LOEWENSTEIN, G.; DONOGHUE, T. O. Animal spirits: affective and deliberative processes in economic behavior. Social Science Research Network, v. 2006, p. 1-54, 2004.

MARDIA, K. V. The effect of nonnormality on some multivariate tests and robustness to nonnormality in the linear model. Biometrika, v. 1, i. 58, p. 105-21, 1971.

MARUYAMA, G. Basics of Structural Equation Modeling. Thousand Oaks CA: Sage, 1998.

MEHRABIN, A. A.; RUSSELL, J. A. An approach to environmental psychology. Cambridge: MIT Press, 1974.

MICK, D. G.; DEMOSS, M. Self-gifts: phenomenological insights from four contexts. Journal of Consumer Research, v. 17, p. 677-682, 1990.

MICU, C. C.; CHOWDHURY, T. G. The Effect of Message’s Regulatory Focus and Product Type on Persuasion. The Journal of Marketing Theory and Practice, v. 5, p. 67-82, 2010.

MULAIK, S. A. et al. Evaluation of goodness-of-fit indices for structural equation model. Psychological Bulletin, v. 105, i. 3, p. 430-445, 1989.

NOBLE, C. H.; KUMAR, M. Using product design strategically to create deeper consumer connections. Business Horizons, v. 51, p. 441-450, 2008.

OLIVER, R. L. Satisfaction: a behavioral perspective on the consumer. McGraw Hill, 1997. v. 14.

PALMER, A.; KOENIG-LEWIS, N. Primary and secondary effects of emotions on behavioral intention of theatre clients. Journal of Marketing Management, v. 26, i. 13-14, p. 1201-1217, 2010.

SCHMITT, B. H.; SIMONSON A. Marketing aesthetics: the strategic management of brands, identity and image. New York: Free Press, 1997.

SCHUMACKER, R. E.; LOMAX, R. G. A beginner's guide to structural equation modeling. New Jersey: Lawrence Erbaum, 1996. 
SCHWARZ, N.; CLORE, G. L. Mood, misattribution, and judgments of well-being: Informative and directive functions of affective states. Journal of Personality and Social Psychology, v. 45, p. 513-523, 1983.

SCHWARZ, N.; CLORE, G. L. Social Psychology: Handbook of Basic Principles. [S.l]: [S.n.], 2007.

SHIMP, T. A.; STUART, E. W. The role of disgust as an emotional mediator of advertising effects. Journal of Advertising, v. 33, p. 43-53, 2004.

SOARS, B. Driving sales trough shoppers' sense of sound, sight, smell and touch. International Journal of Retail Distribution Management, v. 37, i. 3, p. 286-298, 2009.

TAI, S. H. C.; FUNG, A. M. C. Application of an environmental psychology model to in-store buying behaviour. International Retail Distribution Consumer Research, v. 7, p. 311-337, 1997.

TUAN PHAM, M. The logic of feeling. Journal of Consumer Psychology, v. 14, p. 360-369, 2004.

VIEIRA, V. A. Visual aesthetics in store environment and its moderating role on consumer intention. Journal of Consumer Behaviour, v. 9, p. 364-380, 2010.

WARD, P.; DAVIES, B. J.; KOOIJMAN, D. Olfaction and the retail environment: examining the influence of ambient scent. Service Business, v. 1, p. 295-316, 2007.

WINKIELMAN, P.; BERRIDGE, K. C. Unconscious Emotion. Current Directions in Psychological Science, v. 13, p. 120-123, 2004.

YALCH, R.; SPANGENBERG, E. Effects of store music on behavior. Journal of Consumer Marketing, v. 7, i. 2, p. 55-63, 1990.

YI, S.; BAUMGARTNER, H. Coping with negative emotions in purchase-related situations. Journal of Consumer Psychology, v. 14, p. 303-317, 2004. 
Como citar este artigo:

ABNT:

LÜBECK, Rafael Mendes et al. Conexão visual e emocional e a intenção de comprar lingerie para mulheres e a moderação do seu estado civil. RACE: Revista de Administração, Contabilidade e Economia, Ed. Unoesc, v. 15, n. 1, p. 251-274, jan./ abr. 2016. Disponível em: <http://editora.unoesc.edu.br/index.php/race>. Acesso em: dia/mês/ano.

APA:

Lübeck, R. M., Hopner, A., Santini, F. O., \& Martins, J. P. C. (2016). Conexão visual e emocional e a intenção de comprar lingerie para mulheres e a moderação do seu estado civil. RACE: Revista de Administração, Contabilidade e Economia, 15(1), 251-274. Recuperado em dia/mês/ano, de http://editora.unoesc.edu.br/index.php/race 
